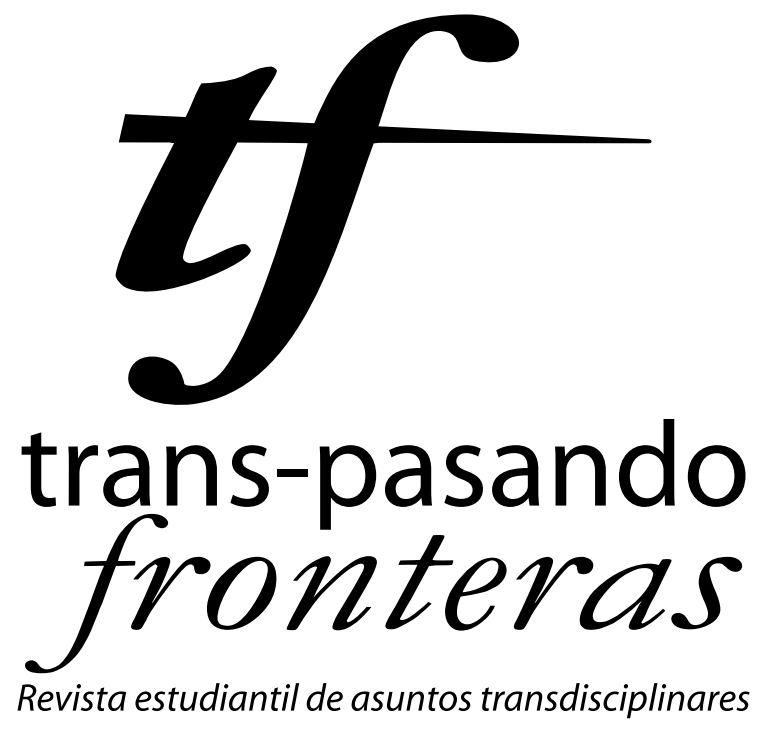

Una publicación de

Estudios

Interdisciplinarios

Jurídicos, Sociales

000 y Humanistas

ICESS

FACULTAD DE

DERECHO Y CIENCIAS

SOCIALES

然 UNIVERSIDAD 


\title{
Crecimiento económico y sostenibilidad ambiental. Un reto compartido por Colombia y China*
}

\author{
Daniela Estrada Nates ${ }^{* *}$ \\ (daniela.estrada@outlook.com)
}

Reporte de caso recibido el 02/05/2013 y aprobado el 23/05/2013.

\begin{abstract}
Como citar este artículo:
ESTRADA NATES, Daniela (2013). "Crecimiento económico y sostenibilidad ambiental, un reto compartido por Colombia y China”. En: Trans-pasando Fronteras, Núm. 3, pp. 143-155. Cali, Colombia: Centro de Estudios Interdisciplinarios, Jurídicos, Sociales y Humanistas (CIES), Facultad de Derecho y Ciencia sociales, Universidad Icesi.
\end{abstract}

\begin{abstract}
Resumen
La contaminación del aire y el agua son fenómenos que han acompañado el crecimiento económico de China y Colombia. Las causas de ambos tipos de contaminación son similares en ambos países, sin embargo la magnitud con la que éstas manifiestan sus consecuencias en términos monetarios y humanos difieren, pues existen características particulares en cada uno de estos dos países. Lo anterior ha sido motivo de una serie de medidas, la cuales aparentemente todavía no logran el efecto esperado, pues aún así el Indicador de Desempeño Ambiental (EPI) calculado por la Universidad de Yale muestra que la situación entre 2008 y 2012 para ambos países ha empeorado. Lo anterior llama la atención e invita a realizar una urgente revisión de las políticas medio ambientales de estas dos naciones.
\end{abstract}

* $\quad$ El siguiente artículo fue premiado en el concurso de ensayos y ponencias en el marco de la XVI Semana Internacional "China: Redefiniendo el orden global” celebrado del 23 al 26 de Abril de 2013 en la Universidad Icesi (Cali, Colombia).

** Estudiante de Economía y negocios internacionales, y Administración de empresas de la Universidad Icesi. 


\section{Palabras claves}

Índice de Desempeño Ambiental, Contaminación medio ambiental, China, Colombia.

\section{Introducción}

El crecimiento económico es una meta deseable puesto que permite alcanzar otro tipo de objetivos; por ejemplo disminuir la tasa de pobreza, mejorar la calidad de vida de las personas, entre otras. Sin embargo, para alcanzar esa meta muchas veces se debe ejercer una presión desmesurada sobre el medio ambiente. Eso implica mayores niveles de deforestación, contaminación y degradación de los ecosistemas, lo cual a su vez puede aumentar los costos del crecimiento económico y causar la desaceleración del mismo, de tal forma que el modelo de desarrollo se vuelve insostenible con el tiempo.

Lo anterior resulta relevante porque tanto China como Colombia son países en desarrollo que han venido experimentando altas tasas de crecimiento en los últimos años (ver Gráfico 1), especialmente China que se ha convertido en la segunda potencia en el mundo. Este crecimiento económico ha sido acompañado de una mejora en indicadores tales como IDH, la tasa de pobreza extrema y los años de educación promedio (ver Anexos: Tabla 5, Tabla 6 y Tabla 7, respectivamente).

\section{Gráfico 1}

Tasas de crecimiento económico Colombia, China y mundial

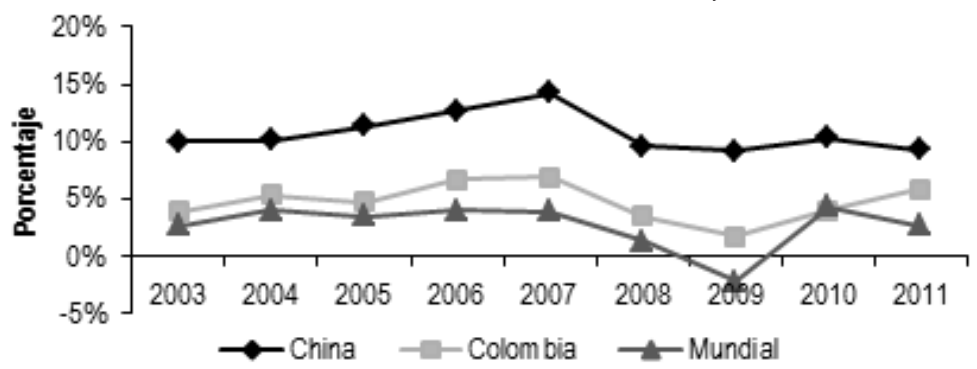

Fuente: Banco Mundial

Sin embargo, noticias tales como: "Pekín se ahoga en medio de la peor nube tóxica de su historia” [China] (El Tiempo, 2013) o "Nuestros océanos están llegando al límite: director de Invemar" [Colombia] (Silva, 2013), evidencian que el agotamiento de recur- 
sos y la contaminación del medio ambiente han acompañado el crecimiento económico y eso ha generado graves problemas. Es por eso que este ensayo tiene como objetivo evidenciar cómo la contaminación del agua y la contaminación del aire han afectado a China y a Colombia, y asimismo busca analizar si las políticas efectuadas para controlar los efectos de la contaminación ambiental han sido efectivas.

Para lograr eso, el ensayo se organiza de la siguiente manera: primero se realizará una descripción general y breve sobre la contaminación del agua y del aire en China y en Colombia, después se presentarán algunos de los efectos que han tenido estos tipos de contaminación. En tercer lugar, se expondrán algunas de las propuestas, normas o acciones más importantes realizadas por los gobiernos para controlar la contaminación ambiental, seguidas de la evaluación de la efectividad de éstas a través del Índice de Desempeño Ambiental, y por último se expondrán las conclusiones.

\section{Contaminación ambiental en China y Colombia}

A continuación, brevemente, se compararán algunos los elementos claves para pensarse la situación relacionada a la contaminación del agua y del aire en China y en Colombia.

\section{Contaminación del agua}

China y Colombia presentan similitudes y diferencias en torno a la contaminación del agua. Por un lado comparten las fuentes de contaminación: urbanización, residuos domésticos, desechos industriales y del sector agropecuario, que son llevados directamente a los ríos sin pasar por plantas de tratamiento. Para el caso colombiano Arjona y Black (s.f.) establecen que el $95 \%$ de las aguas residuales domésticas, el $85 \%$ de las aguas residuales industriales y 95\% de las aguas residuales agrícolas se vierten sin tratamiento alguno.

Otro elemento que comparten es que los principales ríos son los más contaminados. En el caso de China "en el periodo comprendido entre 2001 y 2005, en promedio, alrededor del 54 por ciento de los siete principales ríos no era considerados aptos para el consumo humano". Mientras que para Colombia, el Ministerio de Medio Ambiente ha calculado que la mitad de los recursos hídricos que posee el país tienen problemas de calidad, especialmente los ríos Magdalena, Medellín, Bogotá y Cauca (Beleño, s.f). 


\section{Contaminación del aire}

La contaminación del aire en ambos países proviene principalmente del uso de combustibles fósiles para la producción y el transporte. Sin embargo, en el caso de China la contaminación ha llegado a niveles superiores a los máximos permitidos, y hay presencia de lluvia ácida, fenómeno que no ocurre en Colombia.

Desde 2008, China es el mayor emisor mundial de gases de efecto invernadero en volumen. El aumento de las emisiones se debe principalmente a la dependencia de este país al carbón, pues éste representa más de dos tercios del consumo de energía. Según el informe de Cost of pollution in China (2007) el consumo de energía aumentó un 70\% entre 2000 y 2005, y eso estuvo acompañado de un aumento del $75 \%$ en consumo de carbón. Asimismo, China es la mayor emisora de emisiones de dióxido de azufre del todo el mundo, las cuales causan la lluvia ácida que cae en el 30 por ciento del país.

\section{Efectos de la contaminación ambiental}

La contaminación ambiental afecta de manera directa e indirecta el crecimiento económico La contaminación hídrica afecta la salud, incrementa los costos de tratar el agua y desvaloriza las tierras aledañas a los ríos contaminados, disminuye la actividad pesquera e impacta las áreas acuáticas destinadas a la recreación turística, incrementa los costos de operación y mantenimiento de las hidroeléctricas e impacta negativamente la productividad agrícola, entre otras. La contaminación del aire también afecta la salud, la productividad de las cosechas y la pesca, además daña las infraestructuras y materiales en caso de presentarse lluvia ácida y contribuye al calentamiento global. A continuación se presentan algunos de esos costos valorados en términos monetarios y en vidas humanas.

En términos económicos, se estableció que en 2009, la contaminación y la degradación del medio ambiente representaron el 10.51\% (Zhang, s.f.) del PIB de China. Lo cual significa que ha habido un aumento significativo de los costos puesto que según el estudio de Cost of pollution in China (2007) en 2003 el costo fue de $2.68 \%$ PIB o $5.78 \%$ PIB, dependiendo de la metodología utilizada para su cálculo. En la Tabla 1 se puede observar diferentes fenómenos de la contaminación del agua y sus respectivos costos para este país.

En cuanto a los costos monetarios de la contaminación del aire en China, el Instituto 
Tecnológico de Massachusetts identificó que en 2005 el país perdió 112 mil millones de dólares por disminución de la productividad a causa de los efectos de los contaminantes del aire en la salud (Ee Lyn, 2012). Por otro lado, se ha determinado que aproximadamente 1.2 millones de personas murieron en 2010 debido a la contaminación del aire y que cerca de 190000 se enferman cada año debido al agua contaminada.

Tabla 1

Costos monetarios de la contaminación del agua en China

\begin{tabular}{|l|c|c|c|}
\hline \multicolumn{1}{|c|}{ Elemento } & $\begin{array}{c}\text { Costo (millones de } \\
\text { yuanes) }\end{array}$ & Año & $\begin{array}{c}\text { Por centaje } \\
\text { del PIB }\end{array}$ \\
\hline Mortalidad prematura y morbilidad & 157.300 & 2003 & $1,65 \%$ \\
\hline Escasez de agua asociada a la contaminación & 147.000 & & $1 \%$ \\
\hline Agotamiento del agua subterranea & 50.000 & & \\
\hline Impacto contaminación en pesca comercial & 4.000 & 2003 & \\
\hline Lluvia ácida & 37 & 2003 & \\
\hline Irrigación con agua contaminada & 7 & & \\
\hline
\end{tabular}

Fuente: Cost of pollution in China (2007)

En el caso de Colombia en 2009 el Ministerio de Ambiente reportó la muerte de seis mil personas por causas relacionadas a la contaminación del aire y que el país experimentó una pérdida económica anual de 1.5 billones anuales por cuenta de los efectos que tienen las enfermedades ocasionadas por la contaminación del aire sobre las personas (por ejemplo, ausentismo laboral) . Para el caso de la contaminación del agua, se encontró que en 2008 murieron alrededor de 3000 niños, entre edades de uno a cinco años, debido a que ésta causa proliferación de enfermedades intestinales.

\section{Políticas ambientales: propuestas y evaluación de la efectividad}

La decisión política de realizar acciones para corregir los problemas de contaminación comenzaron para ambos países en el año 1973. La mayoría de las propuestas de estos años estuvieron enfocadas en crear instituciones y normas que regularan la contaminación ambiental. Sin embargo medidas más rigurosas que incluyeran un monitoreo 
continuo y una retroalimentación continua para mejorar códigos inicialmente propuestos sólo se registraron a partir de 2000 (ver Tabla 2 y Tabla 3).

Tabla 2

Propuestas para enfrentar la contaminación ambiental en China

\begin{tabular}{|c|c|c|}
\hline Año & Propuesta & Descripción \\
\hline 1973 & $\begin{array}{l}\text { Primera Conferencia Nacional } \\
\text { sobre Protección del Medio } \\
\text { Ambiente }\end{array}$ & $\begin{array}{l}\text { Primera estrategia nacional de China para proteger el medio } \\
\text { ambiente }\end{array}$ \\
\hline 1979 & $\begin{array}{l}\text { Ley de Protección al Medio } \\
\text { Ambiente (firmada en 1989) }\end{array}$ & - \\
\hline 1982 & El Sexto Plan Quinquenal & El primer plan quinquenal que incorpora protección ambiental \\
\hline 1983 & $\begin{array}{l}\text { Segunda Conferencia Na- } \\
\text { cional sobre Protección del } \\
\text { Medio Ambiente }\end{array}$ & $\begin{array}{l}\text { Protección del medio ambiente se convierte en una política } \\
\text { nacional básica }\end{array}$ \\
\hline 1988 & $\begin{array}{l}\text { Introducción de los permisos } \\
\text { negociables para el control de } \\
\text { la contaminación }\end{array}$ & $\begin{array}{l}\text { Autorizaciones asignadas sobre los niveles de contaminación que } \\
\text { pueden ser negociadas entre los contaminadores locales bajo la } \\
\text { guía de las agencias locales de protección del medio ambiente }\end{array}$ \\
\hline 1993 & $\begin{array}{l}\text { Campaña de Protección del } \\
\text { Medio Ambiente }\end{array}$ & Divulgación al público de información ambiental \\
\hline 2000 & $\begin{array}{l}\text { Revisión de la Ley de Pre- } \\
\text { vención y Control de la Con- } \\
\text { taminación Atmosférica }\end{array}$ & $\begin{array}{l}\text { Revisión de los límites de emisión de los permisos negociables } \\
\text { e introducción de los permisos de emisión de dióxido de azufre }\end{array}$ \\
\hline 2003 & $\begin{array}{l}\text { Tasas impositivas por conta- } \\
\text { minación }\end{array}$ & $\begin{array}{l}\text { Tasas para contaminación de aguas residuales, contaminación del } \\
\text { aire, residuos sólidos y la contaminación acústica. El sistema se } \\
\text { evalúa sobre la cantidad de contaminante presente. }\end{array}$ \\
\hline 2008 & $\begin{array}{l}\text { Ministerio de Protección del } \\
\text { Ambiente }\end{array}$ & $\begin{array}{l}\text { Crea un órgano dentro del gobierno encargado de los asuntos del } \\
\text { Medio Ambiente }\end{array}$ \\
\hline
\end{tabular}

Fuente: Zhang, J. (septiembre de 2012). Delivering Environmentally Sustainable Economic Growth: The Case of China

Además de las propuestas expuestas en las tablas, ambos países también comparten otras medidas tales como beneficios tributarios a las empresas que cumplan con los requisitos expedidos por las normas; divulgaciones sobre reglamentaciones, la contaminación y sus efectos, y las formas cómo se puede aliviar la situación. También cobran tasas por 
Crecimiento económico y sostenibilidad ambiental. Un reto compartido por Colombia y China

contaminación con la intención de que los agentes interioricen las externalidades negativas de sus acciones, y el dinero recaudado es utilizado para mejorar la infraestructura de control y monitoreo de la calidad ambiental. La diferencia en este último punto radica que mientras en Colombia se empezó a implementar en 1997 en China ocurrió seis años después.

Tabla 3

Propuestas para enfrentar la contaminación ambiental en Colombia

\begin{tabular}{|c|c|c|}
\hline Año & Propuesta & Descripción \\
\hline 1973 & Ley 23 & $\begin{array}{l}\text { Prevenir y controlar la contaminación del medio ambiente y bus- } \\
\text { car el mejoramiento, conservación y restauración de los recursos } \\
\text { naturales renovables, para defender la salud y el bienestar de todos } \\
\text { los habitantes del territorio nacional }\end{array}$ \\
\hline 1974 & Decreto Ley 2811 & $\begin{array}{l}\text { Código Nacional de Recursos Naturales Renovables y de Protección } \\
\text { al Medio Ambiente }\end{array}$ \\
\hline 1979 & Ley 99 & $\begin{array}{l}\text { Código Sanitario Nacional (normas, programas y medidas para la } \\
\text { protección del medio ambiente) }\end{array}$ \\
\hline 1984 & Decreto 1594 & $\begin{array}{l}\text { Reglamentación del uso del agua y residuos líquidos, normas en } \\
\text { cuanto a topes de contaminantes }\end{array}$ \\
\hline 1991 & $\begin{array}{l}\text { Constitución Política de } \\
\text { Colombia }\end{array}$ & $\begin{array}{l}\text { Art. } 79 \text { "Todas las personas tienen derecho a gozar de un ambiente } \\
\text { sano. La ley garantizará la participación de la comunidad en las de- } \\
\text { cisiones que puedan afectarlo" y Art. } 80 \text { "Además, deberá prevenir y } \\
\text { controlar los factores de deterioro ambiental, imponer las sanciones } \\
\text { legales y exigir la reparación de los daños causados". }\end{array}$ \\
\hline 1993 & Ley 99 & $\begin{array}{l}\text { Creación del Ministerio del Medio Ambiente y reordenamiento del sec- } \\
\text { tor público encargado de la gestión y conservación del medio ambiente }\end{array}$ \\
\hline 1995 & Decreto 948 & Reglamento de protección y control de la calidad del aire \\
\hline 1997 & $\begin{array}{l}\text { Tasas retributivas por } \\
\text { contaminación hídrica }\end{array}$ & $\begin{array}{l}\text { Consiste en pagar una tarifa por kilogramo cuando se realicen vertimien- } \\
\text { tos puntuales (doméstico, agrícola, industrial) con agua contaminado }\end{array}$ \\
\hline 2005 & $\begin{array}{l}\text { Documento Conpes } \\
3344\end{array}$ & $\begin{array}{l}\text { Lineamientos para la Política de Prevención y Control de la contamina- } \\
\text { ción del aire }\end{array}$ \\
\hline 2008 & Resolución 909 & Normas y estándares admisibles de contaminantes a la atmósfera \\
\hline 2010 & Resolución 650 & Protocolo para el Monitoreo y Seguimiento de la Calidad del Aire \\
\hline
\end{tabular}

Fuente: Política de Prevención y Control de la Contaminación del Aire (2010) y Arjona, F., et. al (2000) 
Debido a las características individuales de cada una de las normas y acciones descritas, se seleccionó el Índice de Desempeño Ambiental' ${ }^{1}$, calculado por la Universidad de Yale, para medir la efectividad global de las propuestas realizadas puesto que este es una medida estándar que permite realizar comparaciones.

Al observar la Tabla 4 se evidencia que las políticas establecidas no están siendo tan efectivas como se esperaría puesto que tanto China como Colombia empeoraron su clasificación. También se puede notar que Colombia ha tenido un mejor desempeño ambiental que China en los tres años. Este desempeño se puede analizar con más profundidad en relación a la contaminación del aire y del agua puesto que el EPI se basa en las medición de dos objetivos fundamentales: el de la salud ambiental que se relaciona con la reducción de las tensiones ambientales para la salud humana y el de vitalidad del ecosistema que se vincula con la protección de los ecosistemas y recursos naturales (ver Anexo: Tablas de la 8 a la 11).

\section{Tabla 4}

Índice de Desempeño Ambiental

\begin{tabular}{|l|c|c|c|c|c|c|}
\hline \multirow{3}{*}{ País } & \multicolumn{3}{|c|}{ Año } \\
\cline { 2 - 7 } & \multicolumn{2}{|c|}{2008} & \multicolumn{2}{c|}{$\mathbf{2 0 1 0}$} & \multicolumn{2}{c|}{2012} \\
\cline { 2 - 7 } & Puesto & Puntos & Puesto & Puntos & Puesto & Puntos \\
\hline China & 105 & 65.1 & 121 & 49 & 116 & 42.2 \\
\hline Colombia & 9 & 88.3 & 10 & 76.8 & 27 & 62.3 \\
\hline
\end{tabular}

Fuente: Politica de Prevención y Control de la Contaminación del Aire (2010) y Arjona, F., et. al (2000)

De ese análisis, se puede deducir que las políticas para proteger la salud humana tanto en el aire como en el agua han resultado ser insuficientes puesto que ambos países sufrieron un empeoramiento significativo en ambos indicadores de salud ambiental, China en 2012 empeoró en un 78\% en el campo de aire respecto a 2010 y Colombia disminuyó en 2012 un 52\% respecto a 2010 en el campo del agua.

Ese mismo comportamiento se evidencia en el ítem de vitalidad ambiental de aire

1 Este clasifica a los países sobre los indicadores de seguimiento de las políticas que cubren tanto la salud pública ambiental y la vitalidad de los ecosistemas. Estos indicadores proporcionan una radiografía de qué tan cercanos están a los países de alcanzar los objetivos de las políticas ambientales establecidas. 
y agua. No obstante la disminución no fue tan significativa como en el caso anterior para Colombia, y de hecho ésta mejoró en el ranking de vitalidad ambiental del aire en 2012 respecto a 2010, lo cual podría estar vinculado con la ejecución del Protocolo para el Monitoreo y Seguimiento de la Calidad del Aire en 2010.

\section{Conclusiones}

En síntesis, China y Colombia están lejos de alcanzar los objetivos propuestos en sus políticas ambientales en relación a la contaminación del aire y el agua a partir de los resultados del análisis de los indicadores EPI. Esa situación resulta preocupante puesto que la tasa de crecimiento de la población de estos países todavía es positiva, lo que significa que a futuro se espera una mayor urbanización y una mayor demanda de recursos que llevaría, de no tomar medidas correctivas efectivas y eficientes, a mayores niveles de contaminación y deterioro ambiental.

Colombia y China pueden guiarse de otros países cuyo desempeño ambiental es mejor, por ejemplo Suiza que ocupó el primer puesto en el ranking EPI 2012. También resulta interesante que China y Colombia compartan experiencias en estos campos puesto que Colombia mejoró en términos de contaminación del aire sobre el medio ambiente, y este fenómeno está afectando gravemente al primero.

Para finalizar, se recomienda investigar cuál ha sido la evolución de la efectividad de la política ambiental en relación a otros tipos de contaminación y deterioro ambiental tales como contaminación de los suelos, pérdida de biodiversidad, entre otros.

\section{Bibliografía}

ACEMOGLU, Daron y James A. Robinson (2012). Por qué fracasan los países. Editorial Planeta Colombiana, Bogotá.

ARJONA, Fabio y Thomas Black (s.f.). El modelo de tasas por contaminación hidrica de Colombia. Consulta realizada en abril de 2013, desde http://goo.gl/ctO8kB (2000). "El caso de Colombia". En: Aplicación de Instrumentos Económicos a la Gestión Ambiental en América Latina y el Caribe, pp. 173-199. CEPAL - Serie Manuales.

BELEÑO, Isis. (s.f.). El 50\% del agua en Colombia es de mala calidad. Consulta realizada en abril de 2013, desde: http://www.unperiodico.unal.edu.co/dper/article/el-50-del-agua-en- 
colombia-es-de-mala-calidad.html

EE LYN, T. (2012). Worsening air pollution costs China dearly: study. Consulta realizada en abril de 2013, http://goo.gl/e1e32i

MADISON, Angus, (2007), Contours of the World Economy, 1-2030 AD. Oxford University Press

NEEDHAM, Joseph y Collin A. Ronan, (1978), The Shorter Science and Civilisation in China: an abridgement of Joseph Needham's Original text. Cambridge and New York: Cambridge University Press

MINISTERIO DE AMBIENTE (s.f.). Tasas Retributivas por Contaminación Hidrica. Consulta realizada en abril de 2013, desde: http://www.minambiente.gov.co// contenido/contenido.aspx? catID $=1296 \&$ conID $=7918$

MINISTERIO DE AMBIENTE, VIVIENDA Y DESARROLLO TERRITORIAL (2010). Política de Prevención y Control de la Contaminación del Aire. Consulta realizada en abril de 2013, desde: http://www.minambiente.gov.co/documentos/normativa/ambiente/politica/ polit_calidad_aire.pdf

QUIROGA, Gloria C. (2007). “China, 30 años de crecimiento económico”. En: Anuario Jurídico y Económico Esculiarense, XLII, pp.463-480

SERVIGÓN CABALLERO, Ab Vicente (2005). "El sistema político y constitucional en China”. En: Revista Jurídica. Ecuador: Universidad Católica de Santiago de Guayaquil

ZHANG, Junjie (s.f.). "Is enviromentally sustainable economic growth possible in China?". En: Asia Society. Consulta realizada en abril de 2013, desde: http://asiasociety.org/policy/environmentally-sustainable-economic-growth-possible-china

(2012). "Delivering Environmentally Sustainable Economic Growth: The Case of China". En: Asia Society. Consulta realizada en abril de 2013, deesde: http://asiasociety.org/files/pdf/Delivering_Environmentally_Sustainable_ Economic_Growth_Case_China.pdf

ZHU, Xiadong, (2012). "Understanding China's Growth: Past, present and future”. En: Journal of Economic Perspectives, volumen 26, Number 4, pp. 103-124

\section{Documentos de prensa}

AMAT, Yamid (2012). “Colombia está al borde de un desastre ambiental: San- 
dra Morelli”. En: El Tiempo. Consulta realizada en abril de 2013, desde: http:// www.eltiempo.com/vida-de-hoy/ecologia/ARTICULO-WEB-NEW_NOTA_INTERIOR-12303681.html

CARACOL NOTICIAS (2008). Miles de niños mueren en Colombia por consumir agua contaminada. Consulta realizada en abril de 2013, desde: http://www.caracol. com.co/noticias/actualidad/miles-de-ninos-mueren-en-colombia-por-consumir-aguacontaminada/20081009/nota/687168.aspx

CARACOL RADIO (2009). En Colombia mueren seis mil personas al año por contaminación del aire, revela Ministerio de Ambiente. Consulta realizada en el abril de 2013, desde: http://goo.gl/co4XGi

EL INFORMADOR (2013). Contaminantes más nocivos en Beijing suben 30\% por año. Consulta realizada en abril de 2013, desde: http://goo.gl/e1e32i

EL TIEMPO (2013). Pekín se ahoga en medio de la peor nube tóxica de su historia. Consulta realizada en abril de 2013, desde:http://goo.gl/HF6otg

SILVA HERRERA, Javier (2013). "Nuestros océanos están llegando al límite': director de Invemar”. En: El Tiempo. Consulta realizada en abril de 2013, desde: http:// www.eltiempo.com/archivo/documento/CMS-12599369

WONG, E. (2013). “Major Air Pollutants Increase in Beijing”. En: New York Times. Consulta realizada en abril de 2013, desde: http://www.nytimes.com/2013/04/04/world/asia/twomajor-air-pollutants-increase-in-china.html?ref=environment\&_r=1\&

Referencia de datos estadísticos

ENVIROMENTAL PERFORMANCE INDEX - EPI (2013). Ranking [años 2008, 2010 y 2012]. Consulta realizada en abril de 2013, desde: http://epi.yale.edu/downloads, Yale University.

- (2013). Country Profile [China y Colombia]. Consulta realizada en abril de 2013, desde: http://epi.yale.edu/downloads, Yale University.

PROGRAMA DE LAS NACIONES UNIDAS PARA EL DESARROLLO - UNDP (s.f.). Índice de Desarrollo Humano- IDH. Recuperado el abril de 2013, de Programa de las Naciones Unidas para el Desarrollo - UNDP: http://hdrstats.undp.org/es/indicadores/103106.html - (s.f.). Población bajo la línea de pobreza de ingresos (\%). Consulta 
realizada en abril de 2013, desde: http://hdrstats.undp.org/es/indicadores/38906.html

WORLD BANK (s.f.). Data Bank GDP growth (annual \%). Consulta realizada en abril de 2013, desde: http://goo.gl/FKhHnz

(2007). Cost of Pollution in China: Economic estimates of physical damages. Consulta realizada en abril de 2013, desde: http://siteresources.worldbank.org/ INTEAPREGTOPENVIRONMENT/Resources/China_Cost_of_Pollution.pdf

\section{Anexos}

Tabla 5

Índice de Desarrollo Humano

\begin{tabular}{|l|c|c|c|c|c|c|c|c|}
\hline \multirow{2}{*}{ País } & \multicolumn{8}{|c|}{ Año } \\
\cline { 2 - 10 } & $\mathbf{2 0 0 0}$ & $\mathbf{2 0 0 5}$ & $\mathbf{2 0 0 6}$ & $\mathbf{2 0 0 7}$ & $\mathbf{2 0 0 8}$ & $\mathbf{2 0 0 9}$ & $\mathbf{2 0 1 0}$ & $\mathbf{2 0 1 1}$ \\
\hline China & 0.59 & 0.637 & 0.65 & 0.662 & 0.672 & 0.68 & 0.689 & 0.695 \\
\hline Colombia & 0.658 & 0.681 & 0.69 & 0.698 & 0.704 & 0.71 & 0.714 & 0.717 \\
\hline
\end{tabular}

Fuente: UNDP

Tabla 6

Población bajo la linea de pobreza \$1.25 USD (PPA)

\begin{tabular}{|l|c|c|c|c|c|c|c|}
\hline \multirow{2}{*}{ País } & \multicolumn{7}{|c|}{ Año } \\
\cline { 2 - 8 } & $\mathbf{2 0 0 0}$ & $\mathbf{2 0 0 5}$ & $\mathbf{2 0 0 6}$ & $\mathbf{2 0 0 7}$ & $\mathbf{2 0 0 8}$ & $\mathbf{2 0 0 9}$ & $\mathbf{2 0 1 0}$ \\
\hline China & & $16.30 \%$ & & & $13.10 \%$ & & \\
\hline Colombia & $17,90 \%$ & $12.70 \%$ & $11 \%$ & $8.80 \%$ & $11.30 \%$ & $9.70 \%$ & $8.20 \%$ \\
\hline
\end{tabular}

Fuente: UNDP

Tabla 7

Años de educación promedio

\begin{tabular}{|l|c|c|c|c|c|c|}
\hline \multirow{2}{*}{ País } & \multicolumn{7}{|c|}{ Año } \\
\cline { 2 - 7 } & $\mathbf{2 0 0 5}$ & $\mathbf{2 0 0 6}$ & $\mathbf{2 0 0 7}$ & $\mathbf{2 0 0 8}$ & $\mathbf{2 0 0 9}$ & $\mathbf{2 0 1 0}$ \\
\hline China & 7.10 & 7.20 & 7.30 & 7.40 & 7.40 & 7.50 \\
\hline Colombia & 6.70 & 6.80 & 7.00 & 7.10 & 7.20 & 7.30 \\
\hline
\end{tabular}

Fuente: UNDP 


\section{Tabla 8}

Índice de Desempeño Ambiental - Ítem Salud Ambiental Aire

\begin{tabular}{|l|c|c|c|}
\hline \multirow{2}{*}{ País } & \multicolumn{3}{|c|}{ Año } \\
\cline { 2 - 4 } & $\mathbf{2 0 0 8}$ & $\mathbf{2 0 1 0}$ & $\mathbf{2 0 1 2}$ \\
\hline China & - & 70.01 & 19.7 \\
\hline Colombia & - & 90.12 & 64.7 \\
\hline
\end{tabular}

Fuente: EPI- Universidad de Yale

Tabla 9

Índice de Desempeño Ambiental - Ítem Salud Ambiental Agua

\begin{tabular}{|l|c|c|c|}
\hline \multirow{2}{*}{ País } & \multicolumn{3}{|c|}{ Año } \\
\cline { 2 - 4 } & $\mathbf{2 0 0 8}$ & $\mathbf{2 0 1 0}$ & $\mathbf{2 0 1 2}$ \\
\hline China & - & 40.07 & 30.2 \\
\hline Colombia & - & 81.62 & 39.2 \\
\hline
\end{tabular}

Fuente: EPI- Universidad de Yale

Tabla 10

Índice de Desempeño Ambiental - Ítem Vitalidad Ambiental Aire

\begin{tabular}{|l|c|c|c|}
\hline \multirow{2}{*}{ País } & \multicolumn{3}{|c|}{ Año } \\
\cline { 2 - 4 } & $\mathbf{2 0 0 8}$ & $\mathbf{2 0 1 0}$ & $\mathbf{2 0 1 2}$ \\
\hline China & 44.9 & 30.19 & 18.2 \\
\hline Colombia & 98.3 & 47.77 & 61.1 \\
\hline
\end{tabular}

Fuente: EPI- Universidad de Yale

Tabla 11

Índice de Desempeño Ambiental - Ítem Vitalidad Ambiental Agua

\begin{tabular}{|l|c|c|c|}
\hline \multirow{2}{*}{ País } & \multicolumn{3}{|c|}{ Año } \\
\cline { 2 - 4 } & $\mathbf{2 0 0 8}$ & $\mathbf{2 0 1 0}$ & $\mathbf{2 0 1 2}$ \\
\hline China & 69.6 & 65.95 & 12.2 \\
\hline Colombia & 74.9 & 69.3 & 53.4 \\
\hline
\end{tabular}

Fuente: EPI- Universidad de Yale 